\title{
ALTERAÇÕES DO OLFATO NA DOENÇA DE PARKINSON
}

\author{
Lucas Barasnevicius Quagliato', Maura Aparecida Viana², \\ Elizabeth Maria Aparecida Barasnevicius Quagliato ${ }^{4}$, Samuel Simis ${ }^{3}$
}

\begin{abstract}
RESUMO - Objetivo: Caracterizar o comprometimento olfatório em 50 pacientes com doença de Parkinson (DP) utilizando o teste de identificação de 12 cheiros da Universidade de Pensilvânia (TICUP), comparando-os com 76 indivíduos normais e associá-lo ao quadro clínico e epidemiológico. Método: Os pacientes foram avaliados na fase "on" com as escalas unificada da doença de Parkinson (UPDRS), Hoehn e Yahr e TICUP e o grupo controle com o TICUP. Resultados: A média geral do número de acertos foi 5,7 nos parkinsonianos e 9 nos controles, com pontuação menor nos que apresentaram como sintoma inicial tremor e naqueles que atualmente apresentavam tremor, rigidez e bradicinesia. A idade e o estágio da DP correlacionaram-se negativamente com o número de acertos, não havendo correlação da perda olfatória com idade de início do quadro e pontuação da UPDRS. Conclusão: Apresentaram comprometimento olfatório $80 \%$ dos pacientes com DP, sendo essa avaliação ferramenta importante no diagnóstico diferencial.
\end{abstract}

PALAVRAS-CHAVE: olfato, doença de Parkinson, UPSIT.

\begin{abstract}
Olfactory dysfunction in Parkinson's disease
ABSTRACT - Objective: To characterize the olfactory dysfunction in 50 Parkinson's disease (PD) patients with the University of Pennsylvania 12 smell identification test (UPSIT), establishing a comparison with 76 agematched healthy controls, and associate with clinical and epidemiologic picture. Method: The PD group was evaluated in phase "on" through United Parkinson's disease rating scale, UPSIT, and Hoehn and Yahr stage and the control group with the UPSIT. Results: The mean UPSIT score was 5.7 in PD patients and 9 in the control group. Patients that presented initially resting tremor and those that currently have tremor, rigidity and bradykinesia had a significant lower scores. There were negative correlation between patients age and PD stage with the UPSIT scores. There were no correlation between olfactory scores, age at the initial PD symptoms and disease duration. Conclusion: Among PD patients $80 \%$ had olfactory deficit and, therefore, smell evaluation may be a tool to make PD differential diagnosis.
\end{abstract}

KEY WORDS: olfaction, Parkinson's disease, UPSIT.

A doença de Parkinson (DP) tem sido considerada afecção predominantemente motora, baseandose seu diagnóstico na presença de tremor em repouso, rigidez, bradicinesia e, na fase avançada, alteração dos reflexos posturais. Há mais de duas décadas são descritas na DP disfunções sensoriais com intensidades variáveis. Olfação, sensibilidade visual ao contraste, percepção visual de cores, propriocepção e controle motor podem apresentar anormalidades, mas, devido à prevalência variável, algumas dessas alterações não preenchem os requisitos de um marcador precoce da $D P^{1}$. A disfunção olfatória é um dos sinais mais prevalentes na DP, ocorrendo em 70 a $90 \%$ dos casos, embora apenas $28 \%$ dos pacientes relatem essa queixa. Observam-se alterações de discriminação, identificação e limiar olfatório' ${ }^{1}$. A hiposmia é um dos sinais que pode anteceder os sintomas motores da DP2 . Pesquisas utilizando o SPECT com beta CIT (I123) e 99mTc TRODAT-1 relacionam a hiposmia ao decréscimo do transporte estriatal da dopamina, atribuindo aos testes olfatórios valor de diagnóstico pré-clínico $0^{3,4}$. Observa-se aumento do limiar olfatório em $97 \%$ dos pacientes com distúrbio comportamental do sono REM, hoje também considerado um indicador pré sintomático da DP, estimando-se que $50 \%$ desses pacientes apresentem posteriormente $\mathrm{DP}^{5}$. Da-

Departamento de Neurologia da Faculdade de Ciências Médicas da Pontifícia Universidade Católica de São Paulo, Sorocaba SP, Brasil: ${ }^{1}$ Estudante da graduação em Medicina; ${ }^{3}$ Professor Dr. Departamento de Neurologia; ${ }^{2}$ Médica contratada; ${ }^{4}$ Profa. Dra. Coordenadora do Grupo de Estudo dos Transtornos do Movimento da Universidade Estadual de Campinas SP, Brasil. Estudo financiado com bolsa de Iniciação Científica do CNPq.

Recebido 21 Setembro 2006, recebido na forma final 24 Janeiro 2007. Aceito 24 Março 2007.

Dra. Elizabeth Maria Aparecida Barasnevicius Quagliato - Neurologia FCM-Unicamp - Caixa Postal 6111 - $13083-970$ Campinas SP - Brasil. E-mail: bethbara@yahoo.com 
dos recentes de um estudo prospectivo com familiares em primeiro grau de pacientes com DP esporádica, correlacionam a hiposmia sem etiologia definida com risco de pelo menos $13 \%$ de desenvolver $\mathrm{DP}^{6}$. A disfunção olfatória tem alta prevalência na fase pré sintomática da DP, não se modifica com as medicações antiparkinsonianas, nem sofre influência das fases "on" e "off"7. Na DP cerca de 60 a $70 \%$ dos neurônios da substância negra compacta (SNc) degeneram antes que se possa estabelecer o diagnóstico clínico. A identificação precoce de pacientes sob risco, portanto, deve ser crucial para que uma terapia protetora tenha sucesso.

A DP esporádica envolve múltiplos sistemas, resultando de alterações em alguns neurônios susceptíveis, manifestando-se clinicamente quando as alterações já atingiram estágio avançado. Seus marcadores biológicos $\propto$-sinucleína imunopositivos - os corpos e neuritos de Lewy - permitem avaliar os alvos desse processo degenerativo. As lesões dos subnúcleos da SNc e das projeções dos seus neurônios, são precedidas e acompanhadas por extenso envolvimento extra-nigral. As lesões do estágio pré- sintomático ocorrem nos núcleos motor dorsal do vago, glossofaríngeo e olfatório anterior. Posteriormente, neurônios menos vulneráveis também são comprometidos, com evolução ascendente e pouca variabilidade interindividual. As lesões do núcleo olfatório anterior vão se tornando mais acentuadas à medida que a doença evolui, enquanto as que ocorrem nas áreas corticais relacionadas a esse núcleo são de intensidade variável. O comprometimento da amígdala, com importantes conexões olfatórias e que se encontra $20 \%$ menor nos pacientes com DP às custas da perda de neurônios do complexo nuclear corticomedial, relaciona-se à hiposmia ${ }^{8}$. 0 intervalo de tempo entre o comprometimento olfatório e o início dos sintomas motores é possivelmente menor que sete anos, como sugerido pelo estudo de pares de gêmeos discordantes para DP ${ }^{9}$. A maioria dos pacientes com DP não tem percepção da sua dificuldade olfatória antes de serem testados e os que já referem essa queixa apresentam as menores pontuações.

O teste breve de identificação de 12 cheiros (TICUP), da Universidade da Pensilvânia, analisa a identificação olfatória, proporciona resultados reprodutíveis e quantifica adequadamente a olfação. Os testes olfatórios constituem uma ferramenta importante no diagnóstico diferencial de tremores com etiologia incerta. A olfação está normal ou discretamente comprometida no tremor essencial, permitindo distingui-lo do ponto de vista nosológico da $\mathrm{DP}^{7}$. A preservação ou leve acometimento do olfato em afec- ções como parkinsonismo vascular, paralisia supranuclear progressiva, degeneração corticobasal e parkinsonismo induzido pelo 1-metil-4-fenil-1,2,3,6-tetra-hydropirina, auxilia no seu diagnóstico diferencial com DP². Na doença do corpos de Lewy a anosmia é achado freqüente, enquanto uma perda moderada do olfato ocorre na doença de Huntington e na atrofia de múltiplos sistemas.

Os objetivos dessa pesquisa foram traduzir para o português a bateria "The Brief Smell Identification Test", avaliar a capacidade de identificação olfatória em 50 pacientes com DP através do TICUP e compará-la com a de 76 indivíduos normais. Também correlacionamos o comprometimento olfatório com estágio da DP (Hohen e Yahr), pontuação na escala unificada da doença de Parkinson (UPDRS) e dados epidemiológicos.

\section{MÉTODO}

O grupo experimental foi constituído de 50 pacientes com DP sem déficit cognitivo (critérios do DSM-IV), procedentes do Ambulatório de Neurologia do Conjunto Hospitalar de Sorocaba (São Paulo) e de uma clínica particular de Campinas (São Paulo). O diagnóstico de DP baseou-se nos critérios do Banco de Cérebros de Londres $^{10}$ e a neuroimagem (RM e TCC) excluiu outros diagnósticos.

Os pacientes assinaram termo de consentimento livre e esclarecido, foram submetidos a anamnese estruturada e examinados na fase "on". Nos dados epidemiológicos constavam idade atual e de início do quadro, sexo, tempo de doença, antecedentes de vida rural, exposição a agrotóxicos, água de poço ou nascente, produtos químicos, tabagismo e utilização de drogas bloqueadoras dopaminérgicas. Foram também relatados sintomas iniciais, atuais, sintomas ortostáticos e pesquisada hipotensão postural. O estágio da doença foi classificado segundo Hohen e Yahr, o quadro clínico com a escala UPDRS e a avaliação olfatória com a bateria de cheiros do TICUP.O grupo-controle foi constituído de 76 indivíduos saudáveis e neurologicamente normais, da mesma faixa etária dos pacientes com DP e que, após ler e assinar o termo de consentimento livre e esclarecido, foram avaliados com o TICUP.

A utilização constante de vasoconstritores nasais, inalações, rinite crônica e perda de olfato secundária a traumatismo craniencefálico foram fatores de exclusão em ambos os grupos.

O TICUP consiste na apresentação de 12 odores (canela, aguarrás, limão, fumaça, chocolate, rosa, solvente de tinta, banana, abacaxi, gasolina, sabonete, cebola), contidos em microcápsulas de polímeros de uréia-formaldeído com 10 a 50 micrômetros, fixadas no canto inferior de 12 páginas. O teste foi auto-administrado, o paciente arranhava a tira que continha o cheiro, aproximava-a da narina, sentia-a e assinalava o odor correspondente dentre as quatro alternativas apresentadas numa folha, auxiliado quando fosse necessário. Mesmo se não sentisse o cheiro, deveria assinalar uma resposta. 
Tabela. Distribuição dos pacientes dos grupos experimental e controle segundo as faixas etárias e análise descritiva do total de acertos no teste da olfação.

\begin{tabular}{|c|c|c|c|c|c|c|c|}
\hline Grupo & Idade & $\mathrm{N}^{\circ}$ & Média & $\mathrm{dp}$ & Mínimo & Mediana & Máximo \\
\hline \multirow[t]{4}{*}{ Parkinson } & $40-50$ & 2 & 7.5 & 0.7 & 7.0 & 7.5 & 8.0 \\
\hline & $51-60$ & 15 & 6.5 & 1.9 & 3.0 & 6.0 & 10.0 \\
\hline & $61-70$ & 18 & 6.3 & 2.4 & 2.0 & 6.5 & 10.0 \\
\hline & $71-80$ & 15 & 3.8 & 2.3 & 0.0 & 4.0 & 8.0 \\
\hline \multirow[t]{4}{*}{ Controle } & $40-50$ & 11 & 9.0 & 1.0 & 8.0 & 9.0 & 11.0 \\
\hline & $51-60$ & 26 & 9.1 & 1.6 & 6.0 & 9.0 & 11.0 \\
\hline & $61-70$ & 25 & 9.0 & 1.1 & 7.0 & 9.0 & 11.0 \\
\hline & $71-80$ & 14 & 8.9 & 1.4 & 6.0 & 9.0 & 12.0 \\
\hline
\end{tabular}

dp, desvio padrão.

Análise estatística - Os dados foram armazenados num banco utilizando o programa Epi-Info (CDC, USA, 2004). Medidas de posição e dispersão para variáveis contínuas e tabelas de freqüências para variáveis categóricas fizeram a análise descritiva. Os testes de Mann-Whitney e de KruskalWallis compararam medidas contínuas ou ordenáveis entre dois e três grupos respectivamente. Comparamos através da análise da covariância as pontuações do TICUP obtidas no grupo experimental com o grupo normativo. Os testes qui-quadrado e exato de Fisher verificaram associações ou compararam proporções. O coeficiente de Spearman avaliou a associação linear. O nível de significância adotado foi $5 \%{ }^{11,12}$. Analisamos os dados com o programa SAStatistical Analysis System for Windows, versão 8.2. (SAS Institute Inc, 1999-2001, USA). Os valores do TICUP do grupo normativo foram calculados com um desvio padrão e intervalo de confiança de 95\% (média -1,96 dp e média +1,96 dp).

$\mathrm{O}$ projeto de pesquisa e os termos de consentimento foram aprovados pelo Comitê de Ética da Pontifícia Universidade Católica de São Paulo.

\section{RESULTADOS}

O grupo de pacientes com DP foi constituído de 25 homens e 25 mulheres, com média de idade de 64,8 anos (dp 8,8; 44 a 80 anos). O grupo controle contou com 76 indivíduos ( 26 homens), média de idade de 61,0 anos (dp 10,3; 40 a 79 anos). Essa diferença significativa entre as médias das idades $(p=0,0424)$ foi contornada através do estudo das faixas etárias separadamente (teste de Mann-Whitney).

A Tabela e a Figura 1 descrevem a distribuição dos pacientes e controles em cada faixa etária e o número de acertos no TICUP.

A média geral do número de acertos no grupo da DP foi 5,7 (dp 2,4; de 0 a 10, mediana 6) e no grupo controle foi 9 (dp 1,3; de 6 a 12 , mediana 9,0), diferença significativa $(p<0,0001)$.

O número mínimo de acertos no grupo controle foi seis, pontuado por apenas três pessoas, tendo as demais identificado corretamente sete ou mais odores. Dois pacientes com DP apresentaram anosmia, 17

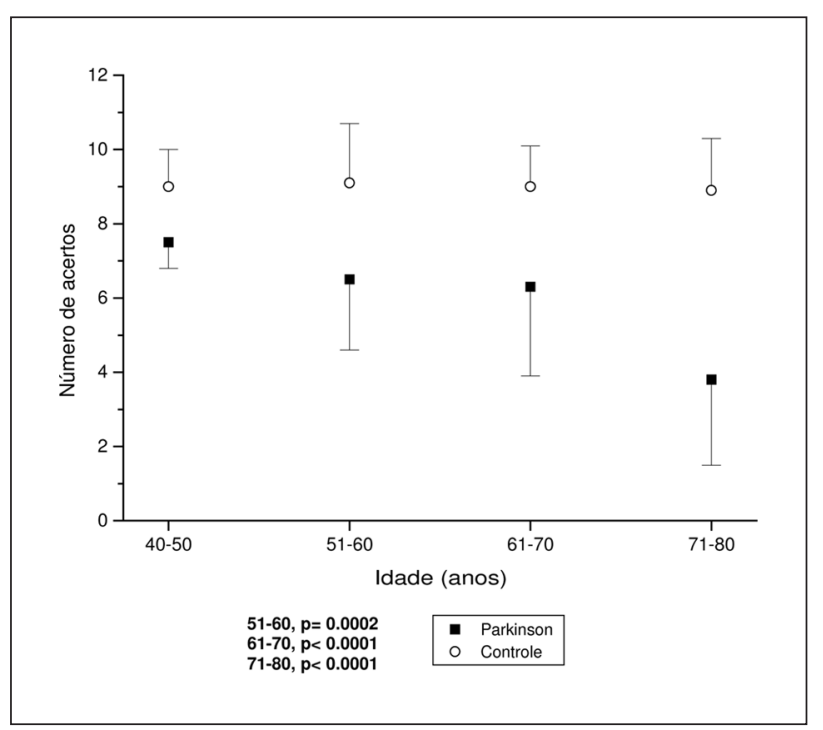

Fig 1. Valor médio e desvio padrão do número de acertos em cada faixa etária e grupo.

acertaram sete ou mais e o maior número de acertos foi 10.

A Figura 2A mostra a distribuição do número de odores identificados corretamente por ambos os grupos e a 2B mostra os valores do TICUP do grupo normativo. Observou-se diferença estatisticamente significativa entre os pacientes com DP e os controles na identificação 11 dos 12 odores ( $p<0,047$ nos 11). Somente o segundo odor (aguarrás) apresentou um número de erros semelhante nos dois grupos ( $\mathrm{p}=$ 0,0858).

Considerando-se um desvio padrão, $80 \%$ dos pacientes com DP apresentaram anormalidade da identificação olfatória, comparados aos controles. Utilizando-se intervalo de $95 \%$ de confiança, $58 \%$ dos pacientes tiveram resultados anormais.

No grupo da DP não ocorreram diferenças na pontuação do TICUP entre os gêneros masculino e feminino (média 5,7 e 5,6, respectivamente; $p=0,7175$ ). 

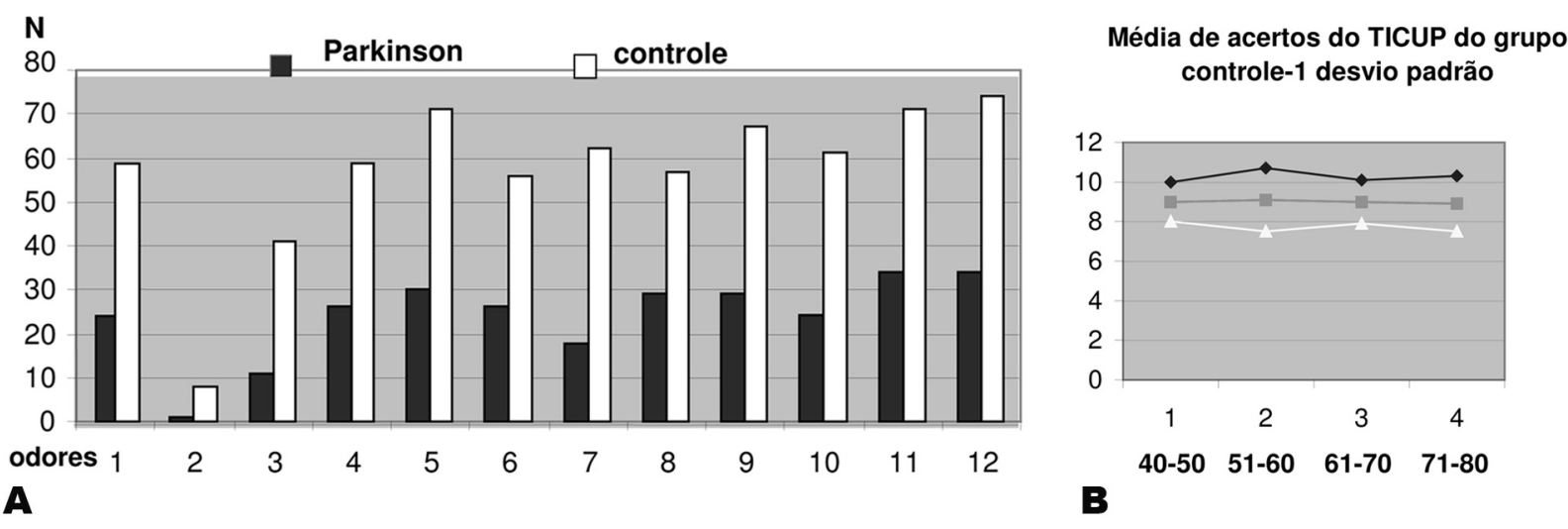

Fig 2. (A) Distribuição do número de acertos em cada odor mostrando diferença significativa em 11 dos 12 odores entre os dois grupos ( $p<0,0449) ; N=$ número de indivíduos que acertou; (B) Acertos do grupo controle no teste da identificação de cheiros da Universidade da Pensilvânia (com 1 desvio padrão).

Também não houve diferença no resultado do TICUP entre os 20 pacientes com antecedente de vida rural $(5,7$ acertos) e os 30 sem esse antecedente (5,6 acertos; $p=0,8107)$. Comparando-se a pontuação do TICUP dos 19 que utilizaram água de poço $(5,6)$ com os que não a utilizaram $(5,7)$, o resultado foi semelhante $(p=0,7019)$. Apenas três pacientes tiveram contato com substâncias químicas ou agrotóxicos, número insuficiente para tecer conclusões estatísticas.

Observou-se correlação negativa do número de acertos no TICUP com o estágio da DP (Hohen e Yahr) (Fig 3; $p=0.0252$ ).

Em relação ao quadro clínico, observou-se média de 5,3 acertos no TICUP nos que apresentaram como sintoma inicial tremor em repouso, significativamente menor que a média dos que não apresentaram tremor inicialmente $(7,6 ; p=0,0383)$. Os 36 pacientes cuja forma clínica atual se caracterizava por tremor, rigidez e bradicinesia apresentaram menor número de acertos (5.2) em comparação com os 14 que não apresentavam atualmente os três sintomas (6.9 acertos; $\mathrm{p}=0,0093$ )

Não se observou diferença significativa da pontuação no TICUP entre os que apresentavam instabilidade postural (11 pacientes, média 4,9 acertos; $\mathrm{p}=0,2672$ ) e sintomas ortostáticos (7 pacientes, média 5,1$)$ e os que não tinham esses sintomas. Apenas um paciente apresentou hipotensão postural.

No grupo controle não houve diferença significativa de pontuação do TICUP entre os cinco que fumavam $(8,4)$ e os 71 que não fumavam $(9,1 ; p=0,2199)$. Os parkinsonianos tabagistas (13) comparados aos não tabagistas (35) apresentaram pontuações semeIhantes (médias de 5,8 e 5,2; $p=0,3611$ ). As pontua-

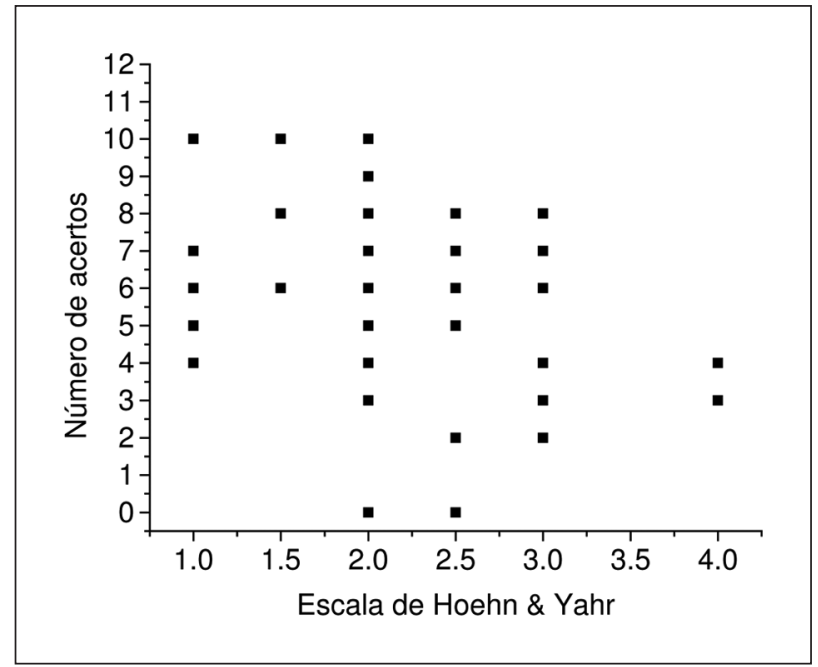

Fig 3. Distribuição do número de acertos em relação ao estágio de Hoenh \& Yahr. Coeficiente de correlação linear de Spearman $=-0,316$.

ções entre os fumantes do grupo controle $(8,4)$ e o da DP $(5,2)$ foram significativamente menores no grupo da DP $(p=0,0130)$.

O coeficiente de Spearman mostrou correlação negativa $(-0,41598)$ entre o número de acertos no TICUP e a idade atual dos pacientes parkinsonianos, mas 0 resultado do TICUP não se correlacionou com a idade no início do quadro $(-0,25799)$, tempo de doença $(-0,27611)$, pontuação das sub-escalas I $(-0,27611)$, II $(-0,08389)$, III $(-0,19432)$ e UPDRS total $(-0,17764)$.

\section{DISCUSSÃO}

Embora raramente sejam avaliados, os distúrbios do olfato e paladar trazem conseqüências como pio- 
ra da qualidade de vida, maior risco de inalar substâncias tóxicas e de ingerir alimentos deteriorados, além de induzir opções alimentares menos saudáveis $^{13}$. O estudo do olfato nas doenças neurodegenerativas tem sido assunto de várias pesquisas, desde que se relacionou disfunção olfatória às doenças de Parkinson e de Alzheimer, que apresentam déficits de olfato indistinguíveis ${ }^{14}$.

Aproximadamente $70 \%$ dos casos de anosmia ou hiposmia crônica são secundários a infecções prévias das vias aéreas superiores, traumatismo craniano e sinusopatias ${ }^{13}$. Desse modo, excluímos indivíduos com esses antecedentes em ambos os grupos.

Cerca de metade da população com idades entre 65 e 80 anos e 3/4 dos indivíduos com mais de 80 anos apresentam disfunção olfatória ${ }^{13}$. 0 grupo normativo dessa pesquisa, com pessoas dos 40 aos 80 anos, permitiu estabelecer comparações com o experimental, demonstrando-se diferença significativa do número de acertos no TICUP em todas as faixas etárias, exceto dos 40 aos 50 anos, possivelmente pelo pequeno número de pacientes. Não observamos no grupo controle diferença significativa do número de acertos no TICUP em relação às faixas etárias, sendo necessário ampliar-se esse grupo melhor descrever, as características da identificação olfatória através do TICUP na nossa população.

A média geral do número de acertos dos pacientes com DP foi 5,7, significativamente menor que a do grupo controle (9), demonstrando a análise dos nossos resultados comprometimento acentuado da identificação olfatória em $58 \%$ dos pacientes com DP. Considerando-se um desvio-padrão, $80 \%$ dos pacientes com DP apresentaram déficit da identificação olfatória quando comparados ao grupo controle, número semelhante aos da literatura ${ }^{1,14}$.

Com a progressão da DP as lesões do núcleo olfatório anterior vão se acentuando, enquanto as das áreas corticais relacionadas a esse núcleo são variáveis. Consoante as publicações de Braak e com o declínio da olfação observado nos idosos, a intensidade da hiposmia observada em nossos pacientes correlacionou-se positivamente com a idade e com o estágio da DP, sendo apenas 4 acertos a média dos pacientes da faixa etária dos 71 aos 80 anos, contra 7,5 nos pacientes com idades entre 40 e 50 anos $^{1,8,15-17}$.

A intensidade da hiposmia não se correlacionou com gênero, gravidade do quadro clínico, idade de início, nem com tempo de duração da DP. A dificuldade de identificar cheiros demonstrou, portanto, ser um fator independente dentro dessa doença multifatorial, conforme já relatado ${ }^{1,17}$.
Pacientes e controles tiveram dificuldade semeIhante para identificar o segundo odor da bateria TICUP (aguarrás), com 1 acerto no grupo DP e 8 no controle. Esse odor deverá ser substituído por não ter sido reconhecido devidamente pelo grupo normativo.

Dentre os 43 pacientes com quadro inicial de tremor em repouso, um dos sinais preditivos do diagnóstico de DP, a média da pontuação do TICUP foi 5,3 , significativamente menor que a média dos que não apresentaram este sintoma inicialmente $(7,6)$. Esse dado aponta para a importância da identificação olfatória no diagnóstico diferencial da etiologia dos tremores. A prevalência da disfunção olfatória é, segundo Hawkes et al., maior que a do tremor e equivalente aos demais sinais da $D P^{18}$.

A hiposmia foi mais acentuada nos 36 pacientes que apresentavam atualmente tremor, rigidez e bradicinesia, comparados aos 14 com quadro motor incompleto (5,2 versus 6,9 acertos). 0 deficit olfatório pode, portanto, ser considerado como um dos sinais cardinais da DP. Instabilidade postural e sintomas ortostáticos, no entanto, não se relacionaram a um pior desempenho no TICUP ${ }^{19}$.

O estudo de Priyadarshi et al. demonstrou aumento do risco de DP associado a fatores ambientais, como residência em área rural, consumo de água de poço e exposição a agrotóxicos ${ }^{20}$. Wright e Keller Byrne relataram aumento do risco para DP aliado ao consumo de água de poço nos primeiros 20 anos de vida, não observando maior risco nos agricultores e nos que usaram agrotóxicos ${ }^{21}$. Em nossa casuística não observamos diferença no resultado do TICUP entre os pacientes com antecedentes de vida rural e utilização de água de poço e os que não apresentaram esses antecedentes, sendo o número dos expostos a pesticidas insuficiente para tecer conclusões do ponto de vista estatístico.

O teste da identificação olfatória, de baixo custo e fácil administração, pode ser um instrumento diagnóstico sensível para pessoas com risco de desenvolver DP. Associando-o à avaliação de outros sinais sutis, como alterações da escrita, fala, depressão, redução do balanço dos braços durante a marcha, pode ser um marcador da fase pré sintomática da DP, como já sugerido por Becker et al. ${ }^{22}$. O estudo da fisiopatologia da disfunção olfatória na DP contribuirá certamente para o desenvolvimento de novas estratégias de tratamento, centradas na detecção pré-clínica e no retardo da progressão da doença ${ }^{23}$.

As peculiaridades do comprometimento olfatório nesse grupo de pacientes sugerem que a disfunção 
olfatória na DP tenha características, desvinculadas dos outros sinais e sintomas. A alta prevalência dessa disfunção torna a avaliação do olfato uma nova ferramenta para se fazer o diagnóstico precoce, além de colaborar para o diagnóstico diferencial das síndromes parkinsonianas.

\section{REFERÊNCIAS}

1. Doty RJ, Bromley SM, Stern MB. Olfactory testing as an aid in the diagnosis of Parkinson's disease: development of optimal discrimination criteria. Neurodegeneration 1995;4:93-97.

2. Katzenschlager R, Lees AJ. Olfaction and Parkinson's syndromes: its role in differential diagnosis. Curr Opin Neurol 2004;17:417-423.

3. Ponsen MM, Stoffers D, Booij J, Van Eck-Smit BL, Wolters ECH, Berendse HW. Idiopathic hyposmia as a preclinical sign of Parkinson's disease. Ann Neurol 2004;56:173-181.

4. Siderowf A, Newberg A, Chou HL, et al. [99mTc]TRODAT-1 SPECT imaging correlates with odor identification in early Parkinson disease. Neurology 2005;64:1716-1720.

5. Stiasny-Kolster K, Doerr Y, Moller JC, et al. Combination of "idiopathic" REM sleep behavior disorder and olfactory dysfunction as possible indicator for alpha-syucleinopathy demonstrated by dopamine transporter FP-CIT-SPECT. Brain 2005;128:126-137

6. Berendse HW, Ponsen MM. Detection of preclinical Parkinson s disease along the olfactory tract .J Neural Transm 2006;70(Suppl):S321-S325.

7. Busenbark KL, Huber SJ, Greer G, Pahwa R, Koller WC. Olfaction function in essential tremor. Neurology 1992;42:1631-1632.

8. Braak H, Del Tredici K, Bratzke H, Hamm-Clement J, Sandmann-Keil $\mathrm{D}$, Rub U . Staging of the intracerebral inclusion body pathology associated with idiopathic Parkinson's disease (preclinical and clinical stages). J Neurol 2002;249:1-5

9. Marras C, Goldman S, Smith A, et al. Smell identification ability in twin pairs discordant for Parkinson's disease.Mov Disord 2005;20:687-693.
10. Hughes AJ, Daniel SE, Kilford L, Lees AJ. Accuracy of clinical diagnosis of idiopathic Parkinson's disease: a clinico-pathological study of 100 cases. J Neurol Neurosurg Psychiatry 1993;55:181-184.

11. Conover WJ. Practical nonparametric statistics. New York: John Wiley \& Sons Inc, 1971.

12. Fleiss JL. Statistical methods for rates and proportions. New York: 2.Ed. John Wiley \& Sons, 1981.

13. Doty RL. Clinical studies of olfaction. Chemical Senses 2005;30(Suppl 1):S207-S209.

14. Raquelle I. Mesholam RI, Moberg PJ, Mah RN, Doty RL. Olfaction in neurodegenerative disease: a meta-analysis of olfactory functioning in Alzheimer's and Parkinson's diseases. Arch Neurol 1998;55:84-90.

15. Braak H, Tredici KD, Rub U, de Vos RAI, Steur ENHJ, Braak E. Staging of brain pathology related to sporadic Parkinson's disease. Neurobiol Aging 2003;24:197-211.

16. Braak H, Ghebremedhin E, Rub U, Bratzke H, Tredici KD. Stages in the development of Parkinson's disease-related pathology. Cell Tissue Res 2004;318:121-134.

17. Doty RL, Deems D, Stellar S. Olfactory dysfunction in Parkinson's disease: a general deficit unrelated to neurologic signs, disease stage, or disease duration. Neurology 1988; 38:1237-1244.

18. Hawkes CH., Shephard BC, Daniel SE. Is Parkinson's disease a primary olfactory disorder? Q J Med 1999;92:473-480.

19. Postuma RB, Lang AE, Massicotte-Marquez J, Montplaisir J. Potential early markers of Parkinson's disease in idiopathic REM sleep behavior disorder. Neurology 2006;66:845-851.

20. Priyadarshi A, Khuder SA, Schaub EA, Priyadarshi SS. Environmental risk factors and Parkinson's disease: a metaanalysis. Environ Res 2001; 86:122-127.

21. Wright JM, Keller-Byrne J. Environmental determinants of Parkinson s disease. Arch Environ Occup Health 2005;60:32-38.

22. Becker G, Muller A, Braune S, et al. Early diagnosis of Parkinson's disease. J Neurol 2002;249(Suppl 3):S40-S48.

23. Harding AJ, Stimson E, Henderson JM, Halliday GM. Clinical correlates of selective pathology in the amygdala of patients with Parkinson's disease. Brain 2002;125:2431-2445. 